\title{
Antifungal Susceptibility Pattern of Candida Isolates Causing Vulvovaginitis in Reproductive Age Women
}

\author{
Pabitra Shrestha ${ }^{1}$, Sunil Mani Pokharel ${ }^{2}$, Anima Shrestha ${ }^{3}$ \\ ${ }^{1}$ Department of Microbiology, Birendra Multiple College, Bharatpur, Chitwan, Nepal \\ ${ }^{2}$ Department of Gynecology and Obstetrics, Bharatpur Hospital, Chitwan, Nepal \\ ${ }^{3}$ Department of Microbiology, Tri-Chandra Multiple Campus, Ghantaghar, Kathmandu, Nepal
}

Corresponding author: Anima Shrestha, Department of Microbiology, Tri-Chandra Multiple Campus, Ghantaghar, Kathmandu, Nepal, Email: animashrestha77@gmail.com, Tel.: 9841248343

\begin{abstract}
Objectives: The study was designed to isolate and identify Candida species from high vaginal swab, and to determine the antifungal susceptibility pattern of Candida spp. among women of reproductive age group i.e. $15-50$ years old.

Methods: High vaginal swabs were processed to isolate Candida species and identified by Gram's stain, germ tube formation test, carbohydrate (glucose, sucrose, maltose, lactose) fermentation tests and antifungal susceptibility test were performed as recommended by Clinical Laboratory Standard Institute.

Results: Out of total 261 samples, 42.1\% (110/261) were found to be culture positive for Candida spp. Candida albicans was the most common $(56.4 \%)$ and among non-albicans, predominate species was C. krusei (19.1\%) isolated from high vaginal swab specimens All the isolates of Candida species were sensitive to Nystatin and Miconazole and resistant to Itraconazole. Fluconazole, Itraconazole and Clotrimazole are widely used drug against vaginal candidiasis but showed high resistance which leads to treatment failure.
\end{abstract}

Conclusion: This study on the infection rate of Candida and its antifungal susceptibility pattern may help in the choice of appropriate therapy in the clinical setting.

Keywords: Vulvovaginal Candidiasis, Vaginal swab, Antifungal Susceptibility , Candida albicans

\section{INTRODUCTION}

Vulvovaginal candidiasis (VVC) or candida vaginitis is a common fungal infection among women of reproductive ages and is caused by overgrowth of Candida species as an opportunistic pathogen. Symptoms of VVC appear when the balance between the normal microorganisms of the vagina is lost and population of C. albicans or other species of Candida becomes larger in relation to the other microorganism populations (Saigal et al. 2011). The most important pathogenic species of Candida are C. albicans, C. glabrata, C. tropicalis, C. parapsilosis and C. krusei (CDC 2010). Although a number of fungal species belonging to the genus Candida can cause acute VVC, Candida albicans is

Date of Submission: August 13, 2020

Published Online: December, 2020 by far the most prevalent etiological agent, particularly for the most severe chronic condition known as recurrent vulvovaginal candidiasis (Cassone 2015).

If untreated they can lead to pelvic inflammatory disease (PID), which can cause long-term sequelae, such as tubal infertility, ectopic pregnancy, reproductive dysfunction and adverse pregnancy outcomes. The most commonly prescribed treatment for vaginal candidiasis has been the topical application of clotrimazole and imidazole antifungal agent. Fluconazole has emerged as the primary treatment option for virtually all forms of susceptible Candida infections in both immune competent and immune compromised hosts. Nystatin

Date of Acceptance: October 13, 2020

DOI: https:/ / doi.org/10.3126/tujm.v7i0.33784 
is known to be effective in vitro against a variety of Candida species (Cavelic et al. 2005).

Nepalese people survive with comparatively very poor health status in South East Asia because of illiteracy, lack of personal hygiene and sanitary knowledge, malnutrition, low economic status and lack of proper techniques in using medical procedures. This justifies people being victimized by many infectious diseases in our country. In this scenario, vaginal candidiasis remains one of the leading causes of the health problem in the country. In this regard, this study aims to investigate the prevalence of vulvovaginal candidiasis and antifungal susceptibility pattern of isolated Candida species from women with the reproductive age group visiting the Department of Gynecology and Obstetrics at Bharatpur Hospital, Chitwan which may be helpful toevaluate the current situation of vaginal candidiasis, effect of the antifungal drugs used for treatment and its management throughout the country.

\section{MATERIALS AND METHODS}

A hospital-based cross-sectional study among reproductive age group (15-49 years) (Mulu et al. 2015) visiting Department of Gynaecology and Obstetrics at Bharatpur Hospital of Chitwan, Nepal was carried out. Two hundred and sixty one individuals (as calculated by Raosoft sample calculator with $5 \%$ of error margin, 95\% confidence interval) were included in the study by convenient sampling method from those willing to give consent and without the condition of HIV/AIDS and/or diabetes, after the study being reviewed and approved by the ethical approval board of Nepal Health Research Council with approval number 167/2015.

Two high vaginal swabs from each of 261 patients collected after preliminary disease diagnosis as VVC by gynecologists were used as samples. The swab samples were then transported to microbiology laboratory and processed as soon as possible according to standard laboratory methods (Cheesbrough 2000).

One of the swabs from each patient, was used for direct microscopy test by Gram staining and another used for culture on two different media- Sabouraud Dextrose agar (HiMedia) supplemented with 0.05 mg/l chloramphenicol and Hichrom agar (HiMedia). Then plates of SDA/chloramphenicol and HiChrom agar were incubated at $35-37^{\circ} \mathrm{C}$ for $24-48$ hours. Identification of isolates was done by appearance of colonies, gram's stain reaction, germ tube formation test and biochemical characteristics (Cheesbrough 2000). Candida species were identified by the color of colonies in HiChrom agar.

Identification of Candida species was followed by antifungal susceptibility test was performed. The test was done by Kirby-Bauer disc diffusion technique according to the procedure described in the Clinical and Laboratory Standard Institute (CLSI-M44-A 2004); a standard dilution of the test isolate was prepared by matching it with $0.5 \mathrm{McF}$ arland turbidity standard and was uniformly swabbed over the antifungal susceptibility assay agar (CLSI 2004). The antifungal discs (Hi-media, Mumbai, India) of fluconazole (25mcg), nystatin (50mcg), miconazole (30 mcg), itraconazole $(10 \mathrm{mcg})$, amphotericin B (10mcg) and clotrimazole $(10 \mathrm{mcg})$ were placed on the medium and incubated at $37^{\circ} \mathrm{C}$ for 48 hours. After incubation period, the zone of inhibition were measured and results were interpreted as per the guidelines given by the Clinical Laboratory Standard Institute (CLSI).

Chi square test was used to evaluate apparent differences for significance at 95\% confidence level. Associations of Candida isolates with different variables were tested. Results were considered significant if $\mathrm{p}$ value was less than 0.05 . Statistical Package for the Social Sciences (SPSS version 21.0) was used for the Chi square test.

\section{RESULTS}

From total of 261 high vaginal swab samples, 110 (42.15\%) were found to be positive for vulvovaginal candidiasis. Out of 110 positive case, the patients of age group 30-35 years were mostly infected27/110 $(24.5 \%)$ and minimum was of age group 15-20 years $5 / 110(4.5 \%)$. However, the relation of infection with age group was not statistically significant (Table 1 ). 
Table 1: Age-wise distribution of participants with growth positive case

\begin{tabular}{cccc}
\hline Age Group & Frequency & Positive Number/Percentage $(\%, \mathbf{n}=\mathbf{1 1 0})$ & $\mathbf{p}$-value \\
\hline $15-20$ & 7 & $5(4.5 \%)$ & \\
$20-25$ & 37 & $22(2 \%)$ & $\mathrm{p}>0.05$ \\
$25-30$ & 42 & $22(20 \%)$ & \\
$30-35$ & 67 & $27(24.5 \%)$ & \\
$35-40$ & 63 & $17(15.5 \%)$ & \\
$40-45$ & 28 & $11(10 \%)$ & \\
$45-49$ & 17 & $6(5.5 \%)$ & \\
Total & 261 & $110(100 \%)$ & \\
\hline
\end{tabular}

Among 72 women who used different types of contraceptives, 29 (26.4\%) were found to be infected. Out of 189 contraceptive non-user women, 81(73.6\%) were found to be infected. The relation between infection and use of contraceptives was not found to be statistically significant (Table 2).

Table 2: Occurrence of VVC with the practice of contraceptive use

\begin{tabular}{ccccc}
\hline \multirow{2}{*}{ Contraceptive used } & Growth positive & Growth negative & \multirow{2}{*}{ Total } & \multirow{2}{*}{ p-value } \\
\cline { 2 - 3 } & Number $(\%)$ & Number $(\%)$ & & \\
User & $29(26.4 \%)$ & $43(28.5 \%)$ & 72 & \\
Non-user & $81(73.6 \%)$ & $108(71.5 \%)$ & 189 & p \\
Total & 110 & 151 & 261 & \\
\hline
\end{tabular}

Five different species of Candida (C. albicans, C. krusei, C. tropicalis, C. parapsilosis and C. dubliniensis) were identified and few isolates were not identified so they were named as Candida species (Figure 1). Candida albicans was the most predominant isolated species. Among 110 infected samples, $82.72 \%$ contained isolates of only one species while the remaining samples were infected with more than species of Candida (Figure 2).

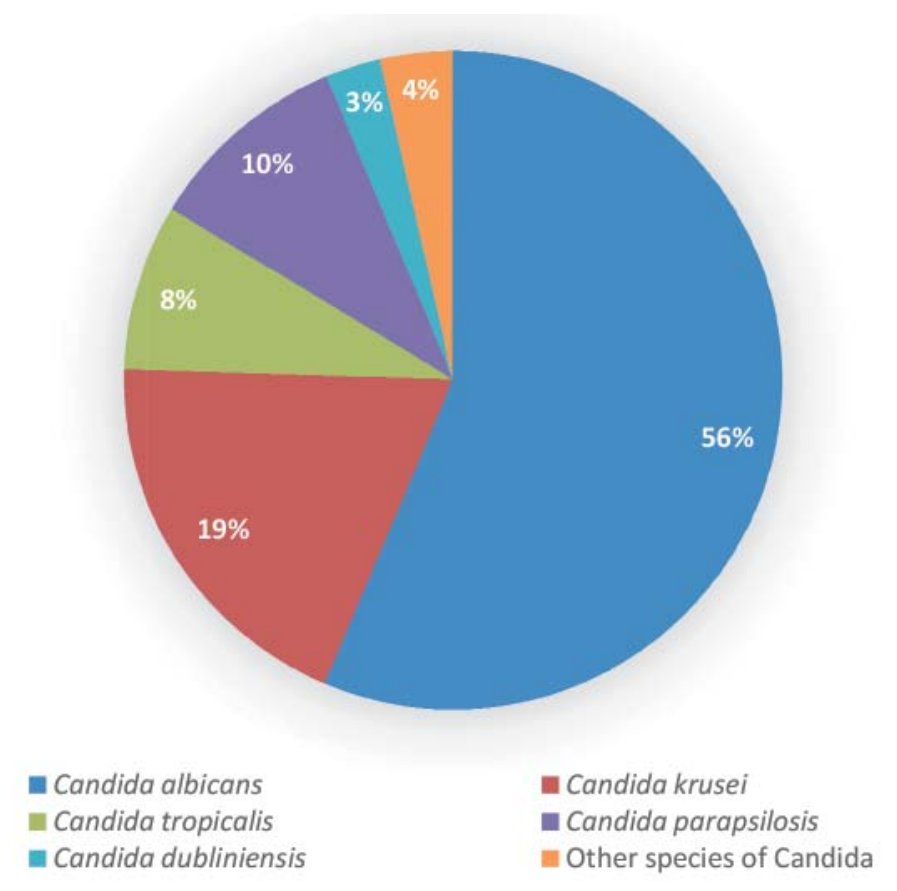

Figure 1: Distribution of Candida species isolated from specimen 


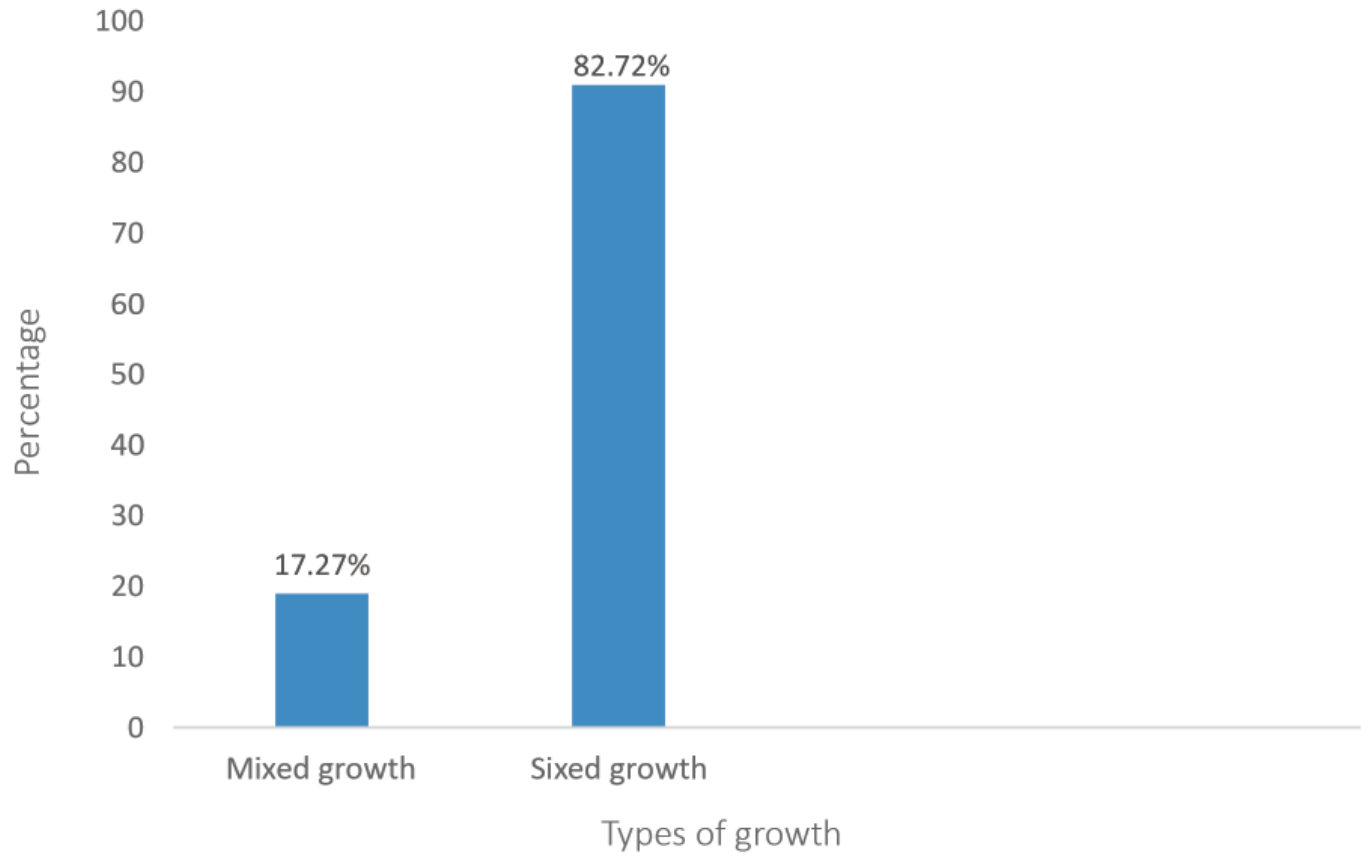

Figure 2: Occurence of single and mixed growth

Antifungal susceptibility test showed all the Candida isolates were susceptible to miconazole and nystatin. Besides, higher numbers of isolates (55\%) were resistant to itraconazole as compared to other antifungals tested. In particular, Candida albicans showed least susceptibility to itraconazole (Table 3).

Table 3: Antifungal susceptibility pattern of isolated Candida species $(\mathbf{n}=\mathbf{1 1 0})$

\begin{tabular}{cccc}
\hline Antifungal agents & Susceptible (\%) & Intermediate (\%) & Resistance (\%) \\
\hline Fluconazole & 49 & 11 & 40 \\
Miconazole & 100 & - & - \\
Nystatin & 100 & 34 & 55 \\
Itraconazole & 11 & 45 & 1 \\
Amphotericin B & 54 & 32 & 15 \\
Clotrimazole & 53 & & - \\
\hline
\end{tabular}

Table 4: Antifungal susceptibility pattern of Candida albicans isolates $(n=62)$

\begin{tabular}{cccc}
\hline Antifungal agents & Susceptible (\%) & Intermediate (\%) & Resistance (\%) \\
\hline Fluconazole & 74.2 & 9.7 & 16.1 \\
Miconazole & 100 & - & - \\
Nystatin & 100 & 50 & 33.9 \\
Itraconazole & 16.1 & 30.6 & 1.6 \\
Amphotericin B & 67.7 & 22.6 & 1.6 \\
Clotrimazole & 75.1 & & - \\
\hline
\end{tabular}

\section{DISCUSSION}

The study showed $42.1 \%$ prevalence of VVC among women with reproductive age which is similar with study carried out by Bello et al. (2002) reported that $41 \%$ prevalence of VVC and other studies as $34.2 \%$ in Ghana and $65 \%$ in South Nigeria. Thus the results may offer a reasonable indication of the prevalence of defined yeast species in vaginitis. In contrast to this study, Rad et al. (2012) concluded that $89.7 \%$ incidence of VVC in Iran whereas Jindal et al. (2007) and Al-aKeel et al. (2013) revealed only $23 \%$ and $22 \%$ prevalent of VVC among women with child bearing 
age respectively. The study conducted by Bohara et al. (2012) at Gynecological Outpatients Department of Tribhuvan University Teaching Hospital (TUTH), Kathmandu, Nepal concluded that out of $62 \%$ RTIs, vaginal candidiasis was found to be $25 \%$.

The rate of infection of Candida species was highest among women of age group 30-35years (24.5\%) and least for 15-20 years group (4.5\%). Shrestha et al. (2011) found the prevalence of vaginitis was common in the age group 20-29 and 30-39 years Manandhar et al. (2005) observed the highest prevalence of vaginitis among the age group 25 to 29 years and least below 20 years age group. The highest prevalence in the age group 30-35 years might be due to the age being the most reproductively active age group and the high sexual exposure at this age and most of the women participated in this study were included from this age group. It is extremely rare before menarche, and its annual incidence increases dramatically towards the end of the second decades of life and peaks over the next two decades. Young women are particularly susceptible to infection because they have fewer antibodies to fight against pathogens (Prasad et al. 2005). The prevalence of vaginitis is low among women with less than 20 years and after 40 years. This might be because during this period, vaginal dryness occurs or it may be because they have less sexual activity. The finding obtained by Crampin et al. (2005) was somewhat different than this study in which the highest prevalent of RTIs among 16 to 22 years was reported.

From the study, it is clear that there is not significant relation in VVC infection and contraceptive use. This type of result is due to the involvement of contraceptive non-user women in higher number. However, Al-akeel et al. (2013) reported that oral-contraceptive users were more likely to developed VVC than non-users.

One of the most important risks for positive Candida species from high vaginal swabs includes recent use of antibiotic/antifungal drugs. This study revealed that there is significant relation in VVC infection and recent drug use. The study carried out by Odysseas et al. (2006) concluded that recent antibiotic use correlated positively with both C. albicans and non-albicans isolates.

The results of this study identified Candida albicans (56.36\%) as the most common species of Candida causing vaginal candidiasis and among non-albicans species, Candida krusei was found to be common species $(19.1 \%)$. This is probably due to the fact that in the general population, C. albicans predominates over other species. The results in this study are consistent with previous study by Akortha et al. (2009) which reported that Candida albicans are the most prevalent with prevalent rates of $64 \%$.

The current findings however contradict the earlier report by Devi and Mahehwari (2014) who reported among the non-albican species, Candida tropicalis was most predominant species followed by C. krusei. - Similar observations were found from other studies (Hollamdia and Young 2003; Spinillo et al. 1994). An overall non albicans percentage were 24, 17 and 32 respectively reported by each of these researchers. Therefore, non-albicans Candida species are emerging significant pathogens (Moran et al. 2003). This variation in reports may be attributed to the different sample sizes used in the studies.

In the study, Miconazole and Nystatin were found to be sensitive against almost all isolates of Candida species. Amphotericin B, Clotrimazole and Fluconazole have been reported as most active antifungal agents. Similar result was revealed by Salehei et al. (2012), vaginal isolates of Candida were most sensitive to Miconazole, Clotrimazole, and Terbinafine, and least sensitive to Econazole, followed by Fluconazole and Ketoconazole. Another study by Vijaya et al. (2011) found that $100 \%$ isolates were sensitive to Amphotericin B, Clotrimazole, Nystatin and Ketaconozole.

\section{CONCLUSION}

Most commonly isolated species in this study was Candida albicans and among non albicans species, Candida krusei was most frequently isolated species. Routine speciation of Candida is becoming increasingly important because vaginal candidiasis due to non albicans species of Candida is increasing nowadays Antifungal susceptibility testing for isolated Candida species from high vaginal swab is important because resistance pattern of Candida species with routinely used antifungal agents is globally increasing. Almost all Candida species isolated in this study were susceptible to Nystatin and Miconazole. Fluconazole, Itraconazole and Clotrimazole are widely used drug against vaginal candidiasis but showed high resistance which may lead to treatment failure. 


\section{ACKNOWLEDGEMENTS}

We would like to acknowledge the medical officers and staffs of Department of Gynaecology and Obstetrics of Bharatpur hospital for their support during sample collection of the study, staffs of clinical laboratory of Bharatpur Hospital and all the participated patients in this study.

\section{CONFLICT OF INTEREST}

The authors declare that they have no competing intersts.

\section{REFERENCES}

Akortha E, Chikwe O and Nwaugo O (2009) Antifungal Resistance among Candida Species from Patients with Genitourinary Tract Infection Isolated in Benin City, Edo estate, Nigeria. African Journal of Microbiology Research 3 (11): 694-699.

Al-akeel RA, El-kersh TA, Al-Sheikh YA and AlAhmadey ZZ (2013) Prevalence and comparison for detection methods of Candida species in vaginal specimens from pregnant and non pregnant Saudi women. African Journal of Microbiology Research 7(1): 56-65.

Bello MD, Gonzalez A, Barnabe C and Larrouy G (2002) First characterization of Candida albicans by Random Amplified Polymorphic DNA method in Nicaragua and comparison of the diagnosis methods for vaginal candidiasis in Nicaraguan women. Men-do-Inst. Osw. Cruz 97(7): 985-989.

Bohara MS, Joshi AB, Lekhak B and Gurung G (2012) Reproductive tract infections among women attending gynaecology outpatient department. International Journal of Infection and Microbiology 1(1): 29-33.

Cassone A (2015) Vulvovaginal Candida albicans infections: pathogenesis, immunity and vaccine prospects. British Journal of Obstetrics and Gynecology 122(6):785-794.

Center for Disease Control and Prevention (2010) Sexually Transmitted Diseases Treatment Guidelines 59(12):62-67.

Cheesbrough M (2000) District Laboratory Practice in Tropical Countries Part 2. $2^{\text {nd }}$ edition. Cambridge University Press pp 129-390.

CLSI, Clinical and Laboratory Standards Institute (2009) Method for antifungal disk diffusion susceptibility testing of yeasts: Approved guideline M44-A, Clinical and Laboratory Standards Institute, Wayne, PA, USA pp 65-74.

Crampin K, Finley K, Gerami-Nejad M, Court H, Gale C, Berman J and Sudbery P (2005) Candida albicans hyphae have a Spitzenkörper that is distinct from the polarisome found in yeast and pseudohyphae. Journal of Cell Science 118: 2935-2947.

DeviSL and Maheshwari M(2014)Speciation of Candida Species Isolated From Clinical Specimens by Using Chrom Agar and Conventional Methods. International Journal of Scientific and Research.4(3): $1-5$

Hollandia J and Young M (2003) Vulvovaginal Carriage of Yeasts Other Than Candida albicans. Sexual Transmission Infections 79(3): 249.

Jindal N, Gill P and Agarwal A (2007) An epidemiological study of vulvovaginal candidiasis in women of childbearing age. Indian Journal of Medical Microbiology 25(2):175-176.

Manandhar R, Sharma J, Pokharel BM, Shrestha BI and Pradhan N (2005) Bacterial vaginosis in Tribhuvan University Teaching Hospital. Journal of Institute of Medicine 27: 14-17.

Moran G, Sullivan D and Coleman D (2003) Emergence of Non-Candida albicans Species as Pathogens in Candidiasis. Calderone RA Ed. ASM Press: Washinton, DC, USA pp 37-54.

Mulu W, Yimer M, Zenebe Y and Abera B (2015) Common causes of vaginal infections and antibiotic susceptibility of aerobic bacterial isolates in women of reproductive age attending at Felegehiwot referral Hospital, Ethiopia: a cross sectional study. BMC Women's Health 15(42): 1-9

Odysseas G, Stavroula B, Evangelos M, Dimitrios H, George K and Evangelia K (2006) Prevalence of clinical vaginal candidiasis in a university hospital and possible risk factors. European Journal of Obstetrics and Gynecology and Reproductive Biology 126(1): 121-125.

Okungbowa F, Isuehuemhen O and Dede A (2003) The Distribution, Frequency of Candida Species in the Genitourinary Tract among Symptomatic individuals in Nigeria cities. Revised Iberoam Microbiology 20: 60-63. 
Pavelic Z, Skalko-Basnet N and Jalsenjak I (2005) Characterisation and in vitro evaluation of bio adhesive liposome gels for local therapy of vaginitis. International Journal of Pharmaceutics 301: 140-148.

PrasadJH, AbrahamSand KurzKM(2005) Reproductive tract infections among young married women in Tamil Nadu, India. International Family Planning Perspectives 31(2): 73-82.

Raad I, Chatzinikolaou I, Chaiban G, Hanna H, Hachem R, Dvorak T, Cook G and Costerton W (2003) In vitro and ex vivo activities of minocycline and EDTA against microorganisms embedded in biofilm on catheter surfaces. Antimicrobial Agents and Chemotherapy 47(11): 3580-3585.

Ragunathan L, Poongothai GK, Sinazer AR, Kannaiyan K, Gurumurthy H, Jaget $\mathrm{N}$ and Kuthalaramalingam S (2014). Phenotypic characterization and antifugal susceptibility pattern to fluconazole in Candida species isolate from vulvovaginal candidiasis in a terteriary care hospital. Jouranl of Clinical and Diagnostic Research 8(5): 1-4.
Saigal S, Bhargava A, Mehra SK and Dakwala F (2011) Identification of Candida albicans by using different media and its association in potentially malignant and malignant lesions. Contemporary Clinical Dentistry 2(3): 188-193.

Salehei Z, Seifi Z and Mahmoudabad AZ (2012) Sensitivity of Vaginal Isolates of Candida to Eight Antifungal Drugs Isolated From Ahvaz, Iran. Jundishapur Journal of Microbiology. 5(4): 574-577.

Shrestha S, Tuladhar NR, Basnyat S, Acharya GP, Shrestha P and Kumar P (2011) Prevalence of vaginitis among pregnant women attending Paropakar Maternity and Women's Hospital, Thapathali, Kathmandu, Nepal. Nepal Medical College Journal 13(4): 293-296.

Spinillo A, Michelone G, Cavanna C, Colonna L, Capuzzo E and Nicola S (1994) Clinical and microbiological characteristics of symptomatic vulvovaginal candidiasis in HIVseropositive women. Genitourinary Medicine 70: 268-272.

Vijaya D, Harsha TR and Nagara T (2011). Candida Speciation Using Chrom Agar. Journal of Clinical and Diagnostic Research 5(4): 755-757. 